\title{
Rigidity Theorem for integral pinched shrinking Ricci solitons
}

\author{
Hai-Ping Fu ${ }^{\mathrm{a}}$, Li-Qun Xiao ${ }^{\mathrm{b}}$ \\ ${ }^{a}$ Department of Mathematics, Nanchang University, Nanchang 330031, P. R. China \\ ${ }^{b}$ Department of management science and Engineering, Nanchang University, Nanchang \\ 330031, P. R. China
}

\begin{abstract}
We prove that an $n$-dimensional, $n \geq 4$, compact gradient shrinking Ricci soliton satisfying a $L^{\frac{n}{2}}$-pinching condition is isometric to a quotient of the round $\mathbb{S}^{n}$, which improves the rigidity theorem given by G. Catino [5]
\end{abstract}

Keywords: Einstein manifold, Ricci soliton, Weyl curvature tensor, Yamabe constant MSC 53C24, 53C20

\section{Introduction}

In this paper we investigate compact gradient shrinking Ricci solitons satisfying a $L^{\frac{n}{2}}$ - pinching condition. Let us recall the concept of Ricci solitons, which was introduced by Hamilton [8] in mid 80's. Let $\left(M^{n}, g\right)$ be an $n$ dimensional, complete, connected Riemannian manifold. A Ricci soliton is a Riemannian metric together with a vector field $\left(M^{n}, g, X\right)$ that satisfies

$$
\operatorname{Ric}+\frac{1}{2} L_{X} g=\lambda g
$$

for some constant $\lambda$. It is called shrinking, steady or expanding Ricci soliton depending on whether $\lambda>0, \lambda=0$ or $\lambda<0$ respectively. If there is a Xiao)

Email addresses: mathfu@126.com (Hai-Ping Fu), xiaoliqun@ncu.edu.cn (Li-Qun

${ }^{1}$ Supported by National Natural Science Foundations of China (11261038, 11361041), Jiangxi Province Natural Science Foundation of China (20132BAB201005). 
smooth function $f$ on $M$ such that $X=\nabla f$, then the equation (1) can be written as

$$
R i c+\nabla^{2} f=\lambda g .
$$

This case is called a gradient (Ricci) soliton. Both equations (1) and (2) can be considered as perturbations of the Einstein equation

$$
\operatorname{Ric}=\lambda g
$$

and reduce to this latter case if $X$ or $\nabla f$ are Killing vector fields. When $X=0$ or $f$ is constant, we call the underlying Einstein manifold a trivial Ricci soliton.

Ricci solitons are an important object in the study of the Ricci flow, since they are self-similar solutions of the flow. They also serve as model cases of various Harnack inequalities for the Ricci flow, which become equalities when the flow consists of Ricci solitons. From the seminal work of Hamilton [8] and Perelman's result [17] that any compact Ricci soliton is necessarily a gradient soliton, it is to see that any compact steady or expanding Ricci soliton must be Einstein [6]. So the classification of complete gradient shrinking solitons plays important roles in the study of the Ricci flow [4]. In dimension three, T. Ivey [13] proved that the only compact shrinking Ricci solitons are quotients of $\mathbb{S}^{3}$ with its standard metric. Dimension four is the lowest dimension where there are interesting examples of shrinking Ricci solitons.

According to the decomposition of the Riemannian curvature tensor, for $n \geq 3$, a locally conformally flat manifold has constant sectional curvature if and only it is Einstein. As a consequence, it follows from the H. Hopf classification theorem that the space forms are isometric to the only complete, simply connected, locally conformally flat, Einstein manifolds. 3-dimensional connected Einstein manifolds are constant curvature spaces. Two isolation theorems of Weyl curvature tensor of positive Einstein manifolds are given in [9, 12], when its $L^{\frac{n}{2}}$-norm is small. The curvature pinching phenomenon plays an important role in global differential geometry. We are interested in $L^{p}$ pinching problems for compact gradient shrinking Ricci solitons.

Now we introduce the definition of the Yamabe constant. Given a compact Riemannian $n$-manifold $M$, we consider the Yamabe functional

$$
Q_{g}: C_{+}^{\infty}(M) \rightarrow \mathbb{R}: f \mapsto Q_{g}(f)=\frac{\frac{4(n-1)}{n-2} \int_{M}|\nabla f|^{2} \mathrm{~d} v_{g}+\int_{M} S f^{2} \mathrm{~d} v_{g}}{\left(\int_{M} f^{\frac{2 n}{n-2}} \mathrm{~d} v_{g}\right)^{\frac{n-2}{n}}}
$$


It follows that $Q_{g}$ is bounded below by Hölder inequality. We set

$$
\mu([g])=\inf \left\{Q_{g}(f) \mid f \in C_{+}^{\infty}(M)\right\} .
$$

This constant $\mu([g])$ is an invariant of the conformal class of $(M, g)$, called the Yamabe constant.

The important works of Schoen, Trudinger and Yamabe showed that the infimum in the above is always achieved (see [1, 15]). There exists an $f \in C_{+}^{\infty}(M)$ satisfying $Q_{g}(f)=\mu([g])$. So, the conformal metric $\tilde{g}=f^{\frac{4}{n-2}} g$ of $g$ has constant scalar curvature $\tilde{S}=\mu([g]) \operatorname{Vol}(\tilde{g})^{-\frac{2}{n}}$, called the Yamabe metric. Choosing a Yamabe metric in the conformal class $[g]$, denoted by $g$, we have the Sobolev inequality

$$
\mu([g])\left(\int_{M} f^{\frac{2 n}{n-2}} \mathrm{~d} v_{g}\right)^{\frac{n-2}{n}} \leq \frac{4(n-1)}{n-2} \int_{M}|\nabla f|^{2} \mathrm{~d} v_{g}+\int_{M} S f^{2} \mathrm{~d} v_{g}, \forall f \in H_{1}^{2}(M) .
$$

The Yamabe constant of a given compact manifold is determined by the sign of scalar curvature [1].

In this note, we obtain the following rigidity theorems.

Theorem 1.1. Let $\left(M^{n}, g\right)(n \geq 4)$ be a complete Einstein $n$-manifold with positive scalar curvature $S$. For $p \geq \frac{n}{2}$, if

$$
\left(\int_{M}|W|^{p}\right)^{\frac{1}{p}}<\left(\frac{\mu([g])}{S}\right)^{\frac{n}{2 p}}\left(\frac{2 S}{C(n) n}\right),
$$

where the constant $C(n)$ is defined in Lemma 2.1, then $M$ is isometric to a quotient of the round $\mathbb{S}^{n}$.

Corollary 1.2. Let $\left(M^{n}, g\right)(n \geq 4)$ be a complete Einstein $n$-manifold with positive scalar curvature $S$. If

$$
\left(\int_{M}|W|^{\frac{n}{2}}\right)^{\frac{2}{n}}<\frac{2 \mu([g])}{C(n) n},
$$

then $M$ is isometric to a quotient of the round $\mathbb{S}^{n}$.

Remark 1.3. Theorem 1.1 has been proved in [7]. It is easy to see from Corollary 1.2 that the pinching constant is better than the one due to 5, , 9, 12]. Theorem 1.1 improves the isolation theorems given by [5, 9, 12, 18]. 
Theorem 1.4. Let $\left(M^{n}, g\right)(n \geq 4)$ be an $n$-dimensional compact shrinking Ricci solitions satisfying

$$
\begin{aligned}
\left(\int_{M}\left|W+\frac{\sqrt{2}}{\sqrt{n}(n-2)} \operatorname{Ric} \otimes g\right|^{\frac{n}{2}}\right)^{\frac{2}{n}} & +\sqrt{\frac{(n-4 \gamma)^{2}(n-1)}{8 \gamma^{2}(n-2)}} \lambda \operatorname{Vol}(g)^{\frac{n}{2}} \\
& <\left(\frac{2}{\gamma}-\frac{1}{\gamma^{2}}\right) \sqrt{\frac{n-2}{32(n-1)}} \mu([g]),
\end{aligned}
$$

where $\gamma=\frac{n+\sqrt{n^{2}+8 n(n-2)^{2}}}{8(n-2)}$. Then $\left(M^{n}, g\right)$ is isometric to a quotient of the round $\mathbb{S}^{n}$.

Remark 1.5. Integrating equation (15), by the definition of $\mu([g])$, we have

$$
\lambda \operatorname{Vol}(g)^{\frac{n}{2}}=\frac{1}{n} \operatorname{Vol}(g)^{\frac{n-2}{n}} \int_{M} S \geq \frac{1}{n} \mu([g]) .
$$

A routine computation gives rise to

$$
\left[\left(\frac{2}{\gamma}-\frac{1}{\gamma^{2}}\right) \sqrt{\frac{n-2}{32(n-1)}}+\left(1-\frac{1}{\gamma}\right) \sqrt{\frac{n-1}{8(n-2)}}\right] \mu([g]) \geq \sqrt{\frac{n-2}{32(n-1)}} \mu([g])
$$

and

$$
\begin{aligned}
& \left(\int_{M}\left|W+\frac{\sqrt{2}}{\sqrt{n}(n-2)} \operatorname{Ric} \otimes g\right|^{\frac{n}{2}}\right)^{\frac{2}{n}}+\sqrt{\frac{(n-4)^{2}(n-1)}{8(n-2)}} \lambda \operatorname{Vol}(g)^{\frac{n}{2}} \\
= & \left(\int_{M}\left|W+\frac{\sqrt{2}}{\sqrt{n}(n-2)} \operatorname{Ric} \otimes g\right|^{\frac{n}{2}}\right)^{\frac{2}{n}}+\sqrt{\frac{(n-4 \gamma)^{2}(n-1)}{8 \gamma^{2}(n-2)}} \lambda \operatorname{Vol}(g)^{\frac{n}{2}} \\
& +\sqrt{\frac{n^{2}(\gamma-1)^{2}(n-1)}{8 \gamma^{2}(n-2)}} \lambda \operatorname{Vol}(g)^{\frac{n}{2}} \\
\geq & \left(\int_{M}\left|W+\frac{\sqrt{2}}{\sqrt{n}(n-2)} \operatorname{Ric} \otimes g\right|^{\frac{n}{2}}\right)^{\frac{2}{n}}+\sqrt{\frac{(n-4 \gamma)^{2}(n-1)}{8 \gamma^{2}(n-2)}} \lambda \operatorname{Vol}(g)^{\frac{n}{2}} \\
& \left.+\left(1-\frac{1}{\gamma}\right) \sqrt{\frac{n-1}{8(n-2)}}\right] \mu([g]) .
\end{aligned}
$$


Hence the integral curvature condition in Theorem 1.4, which holds in every dimension $n \geq 4$, is weaker than the corresponding condition in Theorem 1.4 of [5], holds in every dimension $4 \leq n \leq 6$. We observe that this result apply also to complete (possibly non-compact) gradient shrinking Ricci solitons with positive sectional curvature, since Munteanu and Wang in [16] recently showed that these conditions force the manifold to be compact.

\section{Proof of Theorem 1.1}

In what follows, we adopt, without further comment, the moving frame notation with respect to a chosen local orthonormal frame.

Let $M$ be a complete Einstein $n$-manifold. The decomposition of the Riemannian curvature tensor into irreducible components yield

$$
\begin{aligned}
R_{i j k l}= & W_{i j k l}+\frac{1}{n-2}\left(R_{i k} \delta_{j l}-R_{i l} \delta_{j k}+R_{j l} \delta_{i k}-R_{j k} \delta_{i l}\right) \\
& -\frac{S}{(n-1)(n-2)}\left(\delta_{i k} \delta_{j l}-\delta_{i l} \delta_{j k}\right) \\
= & W_{i j k l}+\frac{S}{n(n-1)}\left(\delta_{i k} \delta_{j l}-\delta_{i l} \delta_{j k}\right),
\end{aligned}
$$

where $R_{i j k l}, W_{i j k l}$ and $R_{i j}$ denote the components of $R m$, the Weyl curvature tensor $W$ and the Ricci tensor Ric, respectively, and $S$ is the scalar curvature.

Now, we compute the Laplacian of $|W|^{2}$.

Lemma 2.1. Let $M$ be a complete Einstein $n$-manifold with scalar curvature S. Then

$$
\triangle|W|^{2} \geq 2|\nabla W|^{2}-2 C(n)|W|^{3}+\frac{4 S}{n}|W|^{2}
$$

where

$$
C(n)=\left\{\begin{array}{l}
\frac{\sqrt{6}}{2}, \quad n=4 \\
\frac{8 \sqrt{10}}{15}, \quad n=5 \\
\frac{4\left(n^{2}+n-4\right)}{\sqrt{(n-1) n(n+1)(n+2)}}+\frac{n^{2}-n-4}{\sqrt{(n-2)(n-1) n(n+1)}}, \quad n \geq 6
\end{array}\right.
$$

Remark 2.2. Lemma 2.1 has been proved in [5, 7]. This estimate is stronger than the one obtained in [5, 7]. 
Proof. By the Ricci identities, we obtain

$$
\begin{aligned}
\triangle|W|^{2}= & 2|\nabla W|^{2}+2\langle W, \triangle W\rangle=2|\nabla W|^{2}+2 W_{i j k l} W_{i j k l, m m} \\
= & 2|\nabla W|^{2}+2 W_{i j k l}\left(W_{i j k m, l m}+W_{i j m l, k m}\right) \\
= & 2|\nabla W|^{2}+4 W_{i j k l} W_{i j k m, l m} \\
= & 2|\nabla W|^{2}+4 W_{i j k l}\left(W_{i j k m, m l}+W_{h j k m} R_{h i l m}\right. \\
& \left.+W_{i h k m} R_{h j l m}+W_{i j h m} R_{h k l m}+W_{i j k h} R_{h m l m}\right) \\
= & 2|\nabla W|^{2}+4 W_{i j k l}\left(W_{h j k m} R_{h i l m}+W_{i h k m} R_{h j l m}\right. \\
& \left.+W W_{i j h m} R_{h k l m}+W_{i j k h} R_{h m l m}\right) \\
= & 2|\nabla W|^{2}+4 W_{i j k l}\left(W_{h j k m} W_{h i l m}+W_{i h k m} W_{h j l m}+W_{i j h m} W_{h k l m}\right. \\
& \left.+W W_{i j k h} W_{h m l m}\right)+\frac{4 S}{n(n-1)} W_{i j k l}\left(W_{l j k i}+W_{i l k j}+W_{i j l k}\right)+\frac{4 S}{n}|W|^{2} \\
= & 2|\nabla W|^{2}+4 W_{i j k l}\left(2 W_{h j k m} W_{h i l m}-\frac{1}{2} W_{i j h m} W_{k l h m}\right)+\frac{4 S}{n}|W|^{2} \\
= & 2|\nabla W|^{2}-4\left(2 W_{i j l k} W_{j h k m} W_{h i m l}+\frac{1}{2} W_{i j k l} W_{h m i j} W_{k l h m}\right)+\frac{4 S}{n}|W|^{2} .
\end{aligned}
$$

Case 1 . When $n=4$, it was proved in [10] that

$$
\left|2 W_{i j l k} W_{j h k m} W_{h i m l}+\frac{1}{2} W_{i j k l} W_{h m i j} W_{k l h m}\right| \leq \frac{\sqrt{6}}{4}|W|^{3} .
$$

Case 2. When $n=5$, Jack and Parker [14] have proved that $W_{i j k l} W_{h m i j} W_{k l h m}=$ $4 W_{i j l k} W_{j h k m} W_{h i m l}$. We consider $W$ as a self adjoint operator on $\wedge^{2} V$. Thus by the algebraic inequality for $m$-trace-free symmetric two-tensors $T$, i.e., $\operatorname{tr}\left(T^{3}\right) \leq \frac{m-2}{\sqrt{m(m-1)}}|T|^{3}$, we obtain

$\left|2 W_{i j l k} W_{j h k m} W_{h i m l}+\frac{1}{2} W_{i j k l} W_{h m i j} W_{k l h m}\right|=\left|W_{i j k l} W_{h m i j} W_{k l h m}\right| \leq \frac{4 \sqrt{10}}{15}|W|^{3}$.

Case 3. When $n \geq 6$, considering $W$ as a self adjoint operator on $S^{2} V$, we have

$$
\begin{aligned}
\mid 2 W_{i j l k} W_{j h k m} W_{h i m l}+ & \frac{1}{2} W_{i j k l} W_{h m i j} W_{k l h m}|\leq 2| W_{i j l k} W_{j h k m} W_{h i m l}\left|+\frac{1}{2}\right| W_{i j k l} W_{h m i j} W_{k l h m} \mid \\
& \leq\left[\frac{2\left(n^{2}+n-4\right)}{\sqrt{(n-1) n(n+1)(n+2)}}+\frac{n^{2}-n-4}{2 \sqrt{(n-2)(n-1) n(n+1)}}\right]|W|^{3} .
\end{aligned}
$$

From (6) and Cases 1,2 and 3, we complete the proof of this Lemma. 
By the Kato inequality $|\nabla W|^{2} \geq \frac{n+1}{n-1}|\nabla| W||^{2}$ (see [2]) and (5), we obtain

$$
|W| \triangle|W| \geq\left.\frac{2}{n-1}|\nabla| W\right|^{2}-C(n)|W|^{3}+\frac{2 S}{n}|W|^{2} .
$$

Let $u=|W|$. Using (6), we compute

$$
\begin{aligned}
u^{\alpha} \triangle u^{\alpha} & =u^{\alpha}\left(\alpha(\alpha-1) u^{\alpha-2}|\nabla u|^{2}+\alpha u^{\alpha-1} \triangle u\right) \\
& =\frac{\alpha-1}{\alpha}\left|\nabla u^{\alpha}\right|^{2}+\alpha u^{2 \alpha-2} u \triangle u \\
& \geq\left[1-\frac{n-3}{(n-1) \alpha}\right]\left|\nabla u^{\alpha}\right|^{2}-C(n) \alpha u^{2 \alpha+1}+\frac{2 S \alpha}{n} u^{2 \alpha},
\end{aligned}
$$

where $\alpha$ is a positive constant.

Proof of Theorem 1.1. When $S>0$, we see from Myers' Theorem that $M$ is compact. So Theorem 1.3 in [11] (see also Proposition 3.1 of [15]) implies that any Einstein metric must be Yamabe, provided it is not conformally flat. We assume that $W$ does not vanish identically. Hence the Einstein metric $g$ is a Yamabe metric in the conformal class $[g]$.

Taking $\alpha=\frac{2 p}{n} \geq 1$. Using the Young's inequality, from (8) we obtain

$$
\begin{aligned}
u^{\alpha} \triangle u^{\alpha} \geq & {\left[1-\frac{n-3}{(n-1) \alpha}\right]\left|\nabla u^{\alpha}\right|^{2}-C(n) \epsilon^{1-\alpha} u^{3 \alpha} } \\
& -\left[C(n)(\alpha-1) \epsilon-\frac{2 S \alpha}{n}\right] u^{2 \alpha} .
\end{aligned}
$$

Setting $w=u^{\alpha}$, we can rewrite (9) as

$$
\begin{aligned}
w \triangle w \geq & {\left[1-\frac{n-3}{(n-1) \alpha}\right]|\nabla w|^{2}-C(n) \epsilon^{1-\alpha} w^{3} } \\
& -\left[C(n)(\alpha-1) \epsilon-\frac{2 S \alpha}{n}\right] w^{2} .
\end{aligned}
$$

By (10), we obtain

$$
\begin{aligned}
w^{\beta} \triangle w^{\beta} \geq & {\left[1-\frac{n-3}{(n-1) \alpha \beta}\right]\left|\nabla w^{\beta}\right|^{2}-C(n) \beta \epsilon^{1-\alpha} w^{2 \beta+1} } \\
& -\beta\left[C(n)(\alpha-1) \epsilon-\frac{2 S \alpha}{n}\right] w^{2 \beta}
\end{aligned}
$$


where $\beta$ is a positive constant. Integrating by parts over $M$, we get

$$
\begin{array}{r}
{\left[2-\frac{n-3}{(n-1) \alpha \beta}\right] \int_{M}\left|\nabla w^{\beta}\right|^{2}-C(n) \beta \epsilon^{1-\alpha} \int_{M} w^{2 \beta+1}} \\
-\beta\left[C(n)(\alpha-1) \epsilon-\frac{2 S \alpha}{n}\right] \int_{M} w^{2 \beta} \leq 0 .
\end{array}
$$

By the Hölder inequality and (12), we have

$$
\begin{array}{r}
{\left[2-\frac{n-3}{(n-1) \alpha \beta}\right] \int_{M}\left|\nabla w^{\beta}\right|^{2}-C(n) \beta \epsilon^{1-\alpha}\left(\int_{M} w^{\frac{2 n \beta}{n-2}}\right)^{\frac{n-2}{n}}\left(\int_{M} w^{\frac{n}{2}}\right)^{\frac{2}{n}}} \\
-\beta\left[C(n)(\alpha-1) \epsilon-\frac{2 S \alpha}{n}\right] \int_{M} w^{2 \beta} \leq 0 .
\end{array}
$$

Set $\frac{1}{\alpha \beta}=\frac{(n-1)\left(1+\sqrt{1-\frac{8(n-3)}{n(n-2) \alpha}}\right)}{n-3}$ and $\epsilon=\frac{2 S}{C(n) n}$. Combining (3) with (13), we get

$$
\left[\left(2-\frac{(n-3)}{(n-1) \alpha \beta}\right) \frac{(n-2) S}{4(n-1)}-C(n) \beta \epsilon^{1-\alpha}\left(\int_{M}|W|^{p}\right)^{\frac{2}{n}}\right]\left(\int_{M} w^{\frac{2 n \beta}{n-2}}\right)^{\frac{n-2}{n}} \leq 0
$$

We choose $\left(\int_{M}|W|^{p}\right)^{\frac{1}{p}}<\left(\frac{\mu([g])}{S}\right)^{\frac{n}{2 p}}\left(\frac{2 S}{C(n) n}\right)$ such that $(14)$ implies $\left(\int_{M} w^{\frac{2 n \beta}{n-2}}\right)^{\frac{n-2}{n}}=$ 0 , that is, $W=0$, i.e., $M$ is Einstein manifold and locally conformally flat manifold. Hence $M$ is isometric to a quotient of the round $\mathbb{S}^{n}$.

\section{Proof of Theorem 1.4}

First, we recall the following well known formulas (for the proof see [9]).

Lemma 3.1. Let $\left(M^{n}, g\right)$ be a gradient Ricci solitons, then the following formulas hold,

$$
\begin{array}{r}
\triangle f=n \lambda-S,(15) \\
\triangle_{f} R_{i k}=\frac{2}{(n-2)(n-1)}\left(S^{2} \delta_{i k}-n S R_{i k}+2(n-1) R_{i j} R_{k}^{j}-(n-1)|R i c|^{2} \delta_{i k}\right) \\
+2 \lambda R_{i k}-2 W_{i j k l} R_{j l},(17)
\end{array}
$$

where the $\triangle_{f}$ denotes the $f$-Laplacian, $\triangle_{f}=\triangle-\nabla_{\nabla f}$. 
Integrating equation (15), one has $n \lambda=\frac{\int_{M} S}{V o l(g)} \geq S_{\text {min }}$. When $S$ gets its minimum, from (16), we get

$$
\triangle S_{\min } \leq \frac{2 S_{\min }}{n}\left(n \lambda-S_{m i n}\right)
$$

This relation, by the strong maximum principle, implies that if $S$ is nonconstant, then it must be positive everywhere, hence $\lambda$ and $\mu([g])$ are positive.

Proof of Theorem 1.4. A simple computation shows the following equation for the $f$-Laplacian of the squared norm of the treceless Ricci tensor.

$$
\begin{aligned}
\frac{1}{2} \triangle_{f}|R i c|^{2}=\frac{1}{2} \triangle|R i c|^{2}-\frac{1}{2}\left\langle\nabla f, \nabla|R i c|^{2}\right\rangle & =|\nabla R i c|^{2}+2 \lambda|R i c|^{2}-2 W_{i j k l} \stackrel{\circ}{R}_{i k} \stackrel{\circ}{R}_{j l} \\
& +\frac{4}{n-2} \stackrel{\circ}{R}_{i j} \stackrel{\circ}{R}_{j k} \stackrel{\circ}{R}_{k i}-\frac{2(n-2)}{n(n-1)} S\left|{ }^{\circ} i c\right|^{2} .(18
\end{aligned}
$$

Using Kato inequality, i.e., $|\nabla|$ Ric $\left.^{\circ}\right|^{2} \leq \mid \nabla$ Ric $^{2}$ at every point where $\mid$ Ric $^{\circ} \mid \neq$ 0 , we have

$$
\begin{array}{r}
|R i c| \triangle|R i c| \geq 2 \lambda \mid \text { Ric }^{2}-2 W_{i j k l} \stackrel{\circ}{R}_{i k} \stackrel{\circ}{R}_{j l}+\frac{4}{n-2} \stackrel{\circ}{R}_{i j} \stackrel{\circ}{R}_{j k} \stackrel{\circ}{R}_{k i} \\
\left.-\frac{2(n-2)}{n(n-1)} S \mid \text { Ric }\left.^{2}\right|^{2}+\frac{1}{2}\langle\nabla f, \nabla| \text { Ric }^{2}\right\rangle .
\end{array}
$$

Set $u=|R i c|$. By (19) and Proposition 2.1 in [5], we compute

$$
\begin{aligned}
u^{\gamma} \triangle u^{\gamma}= & u^{\gamma}\left(\gamma(\gamma-1) u^{\gamma-2}|\nabla u|^{2}+\gamma u^{\gamma-1} \triangle u\right) \\
= & \frac{\gamma-1}{\gamma}\left|\nabla u^{\gamma}\right|^{2}+\gamma u^{2 \gamma-2} u \triangle u \\
\geq & \left(1-\frac{1}{\gamma}\right)\left|\nabla u^{\gamma}\right|^{2}+2 \gamma \lambda u^{2 \gamma}+\gamma\left(-W_{i j k l} \stackrel{\circ}{R}_{i k} \stackrel{\circ}{R}_{j l}+\frac{2}{n-2} \stackrel{\circ}{R}_{i j} \stackrel{\circ}{R}_{j k} \stackrel{\circ}{R}_{k i}\right) u^{2 \gamma} \\
& -\frac{2(n-2)}{n(n-1)} \gamma S u^{2 \gamma}+\frac{1}{2} \gamma u^{2 \gamma-2}\left\langle\nabla f,\left|\operatorname{R}_{i c}\right|^{2} \nabla u^{2}\right\rangle \\
\geq & \left(1-\frac{1}{\gamma}\right)\left|\nabla u^{\gamma}\right|^{2}+2 \gamma \lambda u^{2 \gamma}-\sqrt{\frac{2(n-2)}{n-1}} \gamma\left(|W|^{2}+\frac{8}{n(n-2)} u^{2}\right)^{\frac{1}{2}} u^{2 \gamma} \\
& -\frac{2(n-2)}{n(n-1)} \gamma S u^{2 \gamma}+\frac{1}{2}\left\langle\nabla f, \nabla u^{2 \gamma}\right\rangle .
\end{aligned}
$$


Integrating by parts over $M^{n}$ and using equation (15) it follows that

$$
\begin{array}{r}
0 \geq-\frac{1}{2} \int_{M} u^{2 \gamma} \Delta f+\left(2-\frac{1}{\gamma}\right) \int_{M}\left|\nabla u^{\gamma}\right|^{2}+2 \gamma \lambda \int_{M} u^{2 \gamma} \\
-\sqrt{\frac{2(n-2)}{n-1}} \gamma \int_{M}\left(|W|^{2}+\frac{8}{n(n-2)} u^{2}\right)^{\frac{1}{2}} u^{2 \gamma}-\frac{2(n-2)}{n(n-1)} \gamma \int_{M} S u^{2 \gamma} \\
\geq\left(2-\frac{1}{\gamma}\right) \int_{M}\left|\nabla u^{\gamma}\right|^{2}-\sqrt{\frac{2(n-2)}{n-1}} \gamma \int_{M}\left(|W|^{2}+\frac{8}{n(n-2)} u^{2}\right)^{\frac{1}{2}} u^{2 \gamma} \\
-\frac{n-4 \gamma}{2} \lambda \int_{M} u^{2 \gamma}+\frac{n(n-1)-4(n-2) \gamma}{2 n(n-1)} \int_{M} S u^{2 \gamma} .
\end{array}
$$

For $2-\frac{1}{\gamma}>0$, by the definition of Yamabe constant and (21), we get

$$
\begin{aligned}
0 \geq\left(2-\frac{1}{\gamma}\right) \frac{n-2}{4(n-1)} \mu([g]) & \left(\int_{M} u^{\frac{2 n \gamma}{n-2}}\right)^{\frac{n-2}{n}}-\sqrt{\frac{2(n-2)}{n-1} \gamma} \int_{M}\left(|W|^{2}+\frac{8}{n(n-2)} u^{2}\right)^{\frac{1}{2}} u^{2 \gamma} \\
& -\frac{n-4 \gamma}{2} \lambda \int_{M} u^{2 \gamma}+\frac{2 n+\frac{1}{\gamma} n(n-2)-8(n-2) \gamma}{2 n(n-1)} \int_{M} S u^{2 \gamma} .(22)
\end{aligned}
$$

By Hölder inequality, since $\lambda>0$ and $n-4 \gamma \geq 0$, we obtain

$$
\begin{array}{r}
0 \geq\left[\left(2-\frac{1}{\gamma}\right) \frac{n-2}{4(n-1)} \mu([g])-\sqrt{\left.\frac{2(n-2)}{n-1} \gamma\left(\int_{M}\left(|W|^{2}+\frac{8}{n(n-2)} u^{2}\right)^{\frac{n}{4}}\right)^{\frac{2}{n}}-\frac{n-4 \gamma}{2} \lambda V o l(g)^{\frac{2}{n}}\right]}\right. \\
\left(\int_{M} u^{\frac{2 n \gamma}{n-2}}\right)^{\frac{n-2}{n}}+\frac{2 n+\frac{1}{\gamma} n(n-2)-8(n-2) \gamma}{2 n(n-1)} \int_{M} S u^{2 \gamma} .
\end{array}
$$

Since $W$ is totally trace-free, one has

$$
\mid W+\frac{\sqrt{2}}{\sqrt{n}(n-2)} \text { Ric }\left.\otimes g\right|^{2}=|W|^{2}+\frac{8}{n(n-2)} \mid \text { Ric }^{2}
$$

and the pinching condition (4) implies that $\left(M^{n}, g\right)$ is Einstein. We assume that $W$ does not vanish identically. Hence the Einstein metric $g$ is a Yamabe metric in the conformal class $[g]$, i.e.,

$$
\mu([g])=\operatorname{Vol}(g)^{\frac{n-2}{n}} \int_{M} S .
$$


Moreover, integrating equation (15) one has

$$
\lambda \operatorname{Vol}(g)^{\frac{n}{2}}=\frac{1}{n} \operatorname{Vol}(g)^{\frac{n-2}{n}} \int_{M} S=\frac{1}{n} \mu([g]) .
$$

Hence, the pinching condition (4) implies

$$
\left(\int_{M}|W|^{\frac{n}{2}}\right)^{\frac{2}{n}}<\frac{2}{n \sqrt{2(n-2)(n-1)}} \mu([g]) .
$$

It is sufficient to observe that the pinching constant in (23) is strictly smaller than the one in Corollary 1.2. This completes the proof of Theorem 1.4.

\section{References}

[1] T. Aubin, Some Nonlinear Problems in Riemannian Geometry. Springer-Verlag, Berlin 1998.

[2] S. Bando, A. Kasue and H. Nakajima, On a construction at infinity on manifolds with fast curvature decay and maximal volume growth. Invent. Math. 97 (1989), 313-349.

[3] A. L. Besse, Einstein manifolds. Springer-Verlag, Berlin, 1987.

[4] H. D. Cao, Recent progress on Ricci solitons,arXiv:0908.2006v1 [math.DG], 2009.

[5] G. Cation, Integral pinched shrinking Ricci solitons. arXiv:1509.07416vl [math.DG], 2015.

[6] M. Eminenti, G. La Nave and C. Mantegazza, Ricci solitons: the equation point of view. Manuscript Math. 127 2008, 345-367.

[7] H. P. Fu and L. Q. Xiao, Einstein manifolds with finite $L^{p}$-norm of the Weyl curvature. Submitted to Differ. Geom. Appl. in September 1st 2015 .

[8] R. S. Hamilton, The Ricci flow on surfaces. Mathematics and general relativity, (Santa Cruz,CA, 1986), volume 71 of Contemp. Math., pages 237-262. Am. Math. Soc., 1988. 
[9] E. Hebey and M. Vaugon, Effective $L^{p}$ pinching for the concircular curvature. J. Geom. Anal. 6 (1996), 531-553.

[10] G. Huisken, Ricci deformation of the metric on a Riemannian manifold. J. Differential Geom. 21 (1985), 47-62.

[11] M. Itoh, Yamabe metrics and the space of conformal structures. Intern. J. Math. 2 (1991), 659-671.

[12] M. Itoh and H. Satoh, Isolation of the Weyl conformal tensor for Einstein manifolds. Proc. Jpn. Acad. A 78 (2002), 140-142.

[13] T. Ivey, Ricci solitons on compact threeCmanifolds. Differential Geom. Appl. 3 (1993), 301-307

[14] I. Jack and L. Parker, Linear independence of renormalisation counterterms in curved space-times of arbitrary dimensionality. J. Math. Phys. 28 (1987), 1137-1139.

[15] M. J. Lee and T. H. Parker, The Yamabe problem. Bull. A. M. S. 17 (1987), 37-91.

[16] O. Munteanu and J. Wang, Positively curved shrinking Ricci solitons are compact. arXiv: 1504.07898vl [math.DG], 2015.

[17] G. Perelman, The entropy formula for the Ricci flow and its geometric applications. arXiv:math/0211159vl[math.DG], 2002.

[18] M. Singer, Positive Einstein metrics with small $L_{n / 2}$-norm of the Weyl tensor. Differ. Geom. Appl. 2 (1992), 269-274. 